\title{
Recording arbitrary polarization states on photorefractive media
}

Kaoru Sanaka, Masato Takeuchi, Ryo Moriki, Ryo Suzuki, Yuzuru Takashima

Kaoru Sanaka, Masato Takeuchi, Ryo Moriki, Ryo Suzuki, Yuzuru Takashima, "Recording arbitrary polarization states on photorefractive media," Proc. SPIE 10757, Optical Data Storage 2018: Industrial Optical Devices and Systems, 1075703 (14 September 2018); doi: 10.1117/12.2320022

SPIE Event: SPIE Optical Engineering + Applications, 2018, San Diego, California, United States 


\title{
Recording arbitrary polarization states on photorefractive media
}

\author{
Kaoru Sanaka ${ }^{\mathrm{a}}$, Masato Takeuchi ${ }^{\mathrm{a}}$, Ryo Moriki ${ }^{\mathrm{a}}$, Ryo Suzuki ${ }^{\mathrm{a}}$, and Yuzuru Takashima ${ }^{\mathrm{b}}$ \\ ${ }^{a}$ Department of Physics, Tokyo University of Science, Shinjuku-ku, Tokyo 162-8601, Japan \\ ${ }^{\mathrm{b}}$ College of Optical Sciences, The University of Arizona, 1630 E. Univ. Blvd. Tucson, AZ 85721
}

\begin{abstract}
We propose a new recording scheme by which arbitrary polarization states are stored in volumetric and polarization insensitive photorefractive materials. In the scheme, Stokes parameters of an arbitrary polarization state are recorded as grating strength by using the relation between the interference intensity in recording process and the diffraction efficiency in reading process. We show the experimental setup to retrieve linear, circular, and elliptic polarization states using a typical non-polarization dependent photorefractive media, an iron-doped lithium niobate crystal. The polarization state introduces an additional degree of freedom for storing information, and is useful for expanding the capacity in holographic data storage.
\end{abstract}

\section{INTRODUCTION}

The photorefractive effect offers applications in photonics, such as optical image processing, optical interconnects, parallel optical logic, phase conjugate interferometry, coherent light amplification, and a mass storage media as holographic data storage system. ${ }^{1-3}$ The photorefractive effect is given by a nonlinear change of the effective index of refraction of the medium in the presence of light. When coherent laser beams interfere in a photorefractive media, charge separation occurs due to redistribution of electrons excited from the bright region and diffusion to the dark region of the interference fringes. Spatial redistributed of electrons modulates local index of refraction through an electro-optic effect. The multi-layered structure forms a dynamic volume holograms and offers diffraction effects for input laser beams. The diffraction efficiency is determined by the refractive index numbers of the volume hologram. A volume hologram is formed by the charge redistribution process occurring throughout the volume of the recording material. When we form the volume holograms using photorefractive media, generally the polarization state of signal and reference laser beams should be identical to utilize full dynamic range of the material, consequently overall diffraction efficiency is maximized. Therefore, polarization states have not fully utilized in volume holographic recording except for few attempts to record vector holograms in photo-anisotropic materials has sensitivity for polarization states. ${ }^{4,5}$ Although the polarization recording has been demonstrated in polarization sensitive material, it is desirable to have an option to record polarization states by using polarization insensitive materials commonly available.

Here, we propose a new recording scheme to store and retrieve arbitrary polarization states in polarization independent photorefractive materials, and experimentally demonstrate to record linear, circular and elliptic polarization states on a representative inorganic photorefractive media, iron-doped lithium niobate crystal. The polarization states of object beam are recorded as three holograms while changing polarization states of reference beam so that diffraction efficiencies of the three holograms satisfies.

Further author information: (Send correspondence to K.S. )

K.S.: sanaka@rs.tus.ac.jp, Telephone: 81352288213

Optical Data Storage 2018: Industrial Optical Devices and Systems, edited by Ryuichi Katayama,

Yuzuru Takashima, Proc. of SPIE Vol. 10757, 1075703 - (c) 2018 SPIE

CCC code: $0277-786 X / 18 / \$ 18 \cdot$ doi: $10.1117 / 12.2320022$

Proc. of SPIE Vol. 10757 1075703-1 
For general photorefractive materials, the diffraction efficiency in reading process linearly scales with the interference intensity in recording process under Born approximation for weak volume holograms which is a typical scenario for holographic data storage systems employing large number of multiplexed holograms. Using the proportional relation, we demonstrated recording three Stokes parameters of an arbitrary polarization states on three spatially separated locations of a photorefractive material. Upon read out the Stokes parameters of the recorded polarization states are back calculated by the relation of diffraction efficiencies from the three positions. The proposed recording scheme introduces an additional degree of freedom (DOF) for holographic data storage system in terms of increasing number of multiplexing beyond the geometrical limit in angular multiplexing and/or eliminating mechanical scanning for angular multiplexed volume holographic storages, and eventually incorporated into advanced recording schemes such as amplitude-phase recording and homodyne detections.

\section{THEORY}

\subsection{Representation of polarization states}

Non-depolarized state of polarization can be represented by Jones Vector or Stokes parameters. ${ }^{6-8}$ The Jones Vector of an arbitrary polarization state can be written as

$$
|\varphi\rangle=\cos \left(\frac{\theta}{2}\right)|H\rangle+\mathrm{e}^{i \phi} \sin \left(\frac{\theta}{2}\right)|V\rangle \equiv\left(\begin{array}{c}
\cos \left(\frac{\theta}{2}\right) \\
\mathrm{e}^{i \phi} \sin \left(\frac{\theta}{2}\right)
\end{array}\right) .
$$

Where $|H\rangle \equiv\left(\begin{array}{l}1 \\ 0\end{array}\right)$ and $|V\rangle \equiv\left(\begin{array}{l}0 \\ 1\end{array}\right)$ show the Jones Vector of horizontal and vertical polarization state, respectively. $\theta$ and $\phi$ are the angles to represent the Poincaré sphere surface position as dipicted in Figure 1. In Table 1, representative polarization states are tabulated.

Table 1. Representative polarization states

\begin{tabular}{ccc}
\hline Polarization state & $\theta, \phi$ & Jones Vector \\
\hline \hline $\mathrm{H}$ & $\theta=0$ & $|H\rangle$ \\
$\mathrm{V}$ & $\theta=\pi$ & $|V\rangle$ \\
$\mathrm{D}$ & $\theta=\frac{\pi}{2}, \phi=0$ & $|D\rangle=\frac{1}{\sqrt{2}}(|H\rangle+|V\rangle)$ \\
$\mathrm{A}$ & $\theta=\frac{\pi}{2}, \phi=\pi$ & $|A\rangle=\frac{1}{\sqrt{2}}(|H\rangle-|V\rangle)$ \\
$\mathrm{R}$ & $\theta=\frac{\pi}{2}, \phi=\frac{\pi}{2}$ & $|R\rangle=\frac{1}{\sqrt{2}}(|H\rangle+i|V\rangle)$ \\
$\mathrm{L}$ & $\theta=\frac{\pi}{2}, \phi=-\frac{\pi}{2}$ & $|L\rangle=\frac{1}{\sqrt{2}}(|H\rangle-i|V\rangle)$ \\
\hline
\end{tabular}

When the light is pure polarized and light intensity of any recording polarization state is same, any polarization state can be represented by three Stokes parameters $S_{1}, S_{2}, S_{3}{ }^{7}$ Since three Stokes parameters correspond to the coordinates in Poincaré sphere in Figure 1, the Stokes parameters can be represented by angle $\theta$ and $\phi$ as

$$
\begin{aligned}
& S_{1}=\cos \phi \sin \theta, \\
& S_{2}=\sin \phi \sin \theta, \\
& S_{3}=\cos \theta .
\end{aligned}
$$

Here Stokes parameters are normalized for the unit light intensity as $\sum_{i=1}^{3} S_{i}^{2}=1$. 


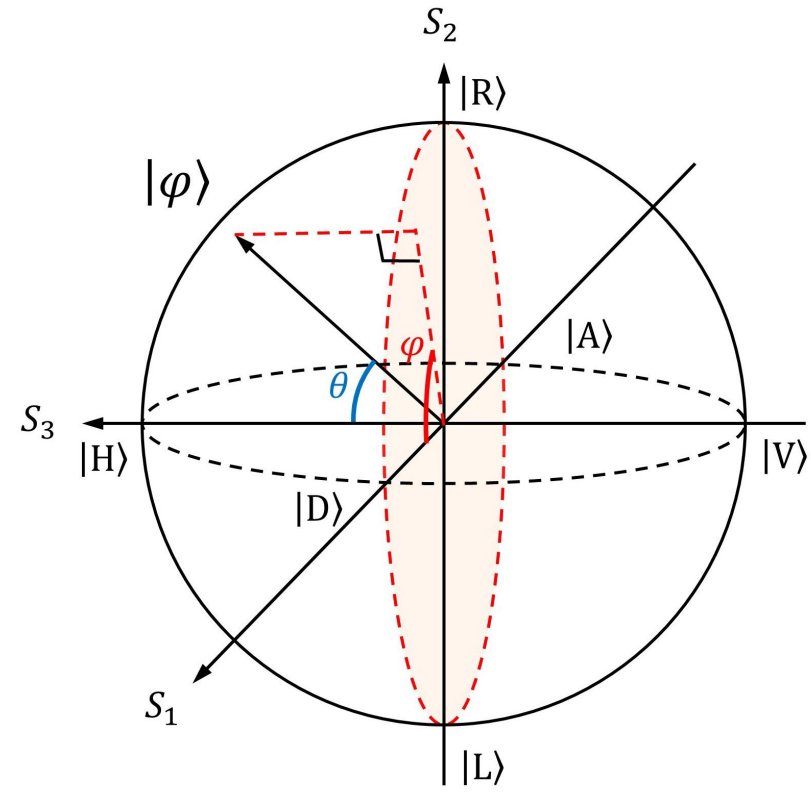

Figure 1. Poincaré sphere and polarization base

\subsection{Method of recording and reading an arbitrary polarization state}

Figure 2 schematically depicts optical setup record an arbitrary polarization state $|\varphi\rangle$ in a photorefractive material. Here we suppose that the material is polarization independent and volumetric. . We prepare for the object beam as a purely polarized light $|\varphi\rangle$ shown in (1), and reference beam as horizontally polarized light $|H\rangle$. The recording process of an arbitrary polarization state $|\varphi\rangle$ is employed by the following three steps.

First, we record the state as fringe modulation intensity between object and reference beam in photorefractive material shown in the Figure 2a. Since the fringe modulation intensity between objective and reference beam is proportional to $|\langle H \mid \varphi\rangle|^{2}$, the intensity $I$ can be written as $I=C|\langle H \mid \varphi\rangle|^{2}$. Here $C$ is a proportional constant. When both objecti and reference beam are in the same state of polarization, the interference intensity becomes maximum and can be written as $I_{\max }=C|\langle H \mid H\rangle|^{2}$. Using (1) and the relation between $I$ and $I_{\max }$, the interference intensity between an arbitrary polarization state of the light $|\varphi\rangle$ and horizontally polarized light $|H\rangle$ can be represented as

$$
I=I_{\max } \cos ^{2}\left(\frac{\theta}{2}\right)
$$

Second, we record the state as fringe modulation intensity by using object beam where a half-wave plate (HWP) is placed as shown in the Figure 2b. A HWP converts the polarization bases of the objective beam as $|H\rangle \rightarrow(|H\rangle+|V\rangle) / \sqrt{2},|V\rangle \rightarrow(|H\rangle-|V\rangle) / \sqrt{2}$. The polarization state $|\varphi\rangle$ is converted into the following state as

$$
\left|\varphi_{\mathrm{HWP}}\right\rangle=\frac{1}{\sqrt{2}}\left\{\cos \left(\frac{\theta}{2}\right)+\mathrm{e}^{i \phi} \sin \left(\frac{\theta}{2}\right)\right\}|H\rangle+\frac{1}{\sqrt{2}}\left\{\cos \left(\frac{\theta}{2}\right)-\mathrm{e}^{i \phi} \sin \left(\frac{\theta}{2}\right)\right\}|V\rangle
$$

Therefore the interference intensity between the objective beam $\left|\varphi_{\mathrm{HWP}}\right\rangle$ and reference beam $|H\rangle$ is shown as

$$
I_{\mathrm{HWP}} \equiv C\left|\left\langle H \mid \varphi_{\mathrm{HWP}}\right\rangle\right|^{2}=\frac{I_{\max }}{2}(1+\cos \phi \sin \theta) .
$$


Third, we record the state with an object beam placing a quarter-wave plate (QWP) shown in the Figure 2c. A QWP converts the polarization bases of the objective beam as $|H\rangle \rightarrow(|H\rangle+i|V\rangle) / \sqrt{2},|V\rangle \rightarrow(i|H\rangle+|V\rangle) / \sqrt{2}$. The polarization state $|\varphi\rangle$ is converted into the following state as

$$
\left|\varphi_{\mathrm{QWP}}\right\rangle=\frac{1}{\sqrt{2}}\left\{\cos \left(\frac{\theta}{2}\right)+i \mathrm{e}^{i \phi} \sin \left(\frac{\theta}{2}\right)\right\}|H\rangle+\frac{i}{\sqrt{2}}\left\{\cos \left(\frac{\theta}{2}\right)-i \mathrm{e}^{i \phi} \sin \left(\frac{\theta}{2}\right)\right\}|V\rangle .
$$

Therefore the interference intensity between the objective beam $\left|\varphi_{Q W P}\right\rangle$ and reference beam $|H\rangle$ is shown as

$$
I_{\mathrm{QWP}} \equiv C\left|\left\langle H \mid \varphi_{\mathrm{QWP}}\right\rangle\right|^{2}=\frac{I_{\mathrm{max}}}{2}(1-\sin \phi \sin \theta) .
$$

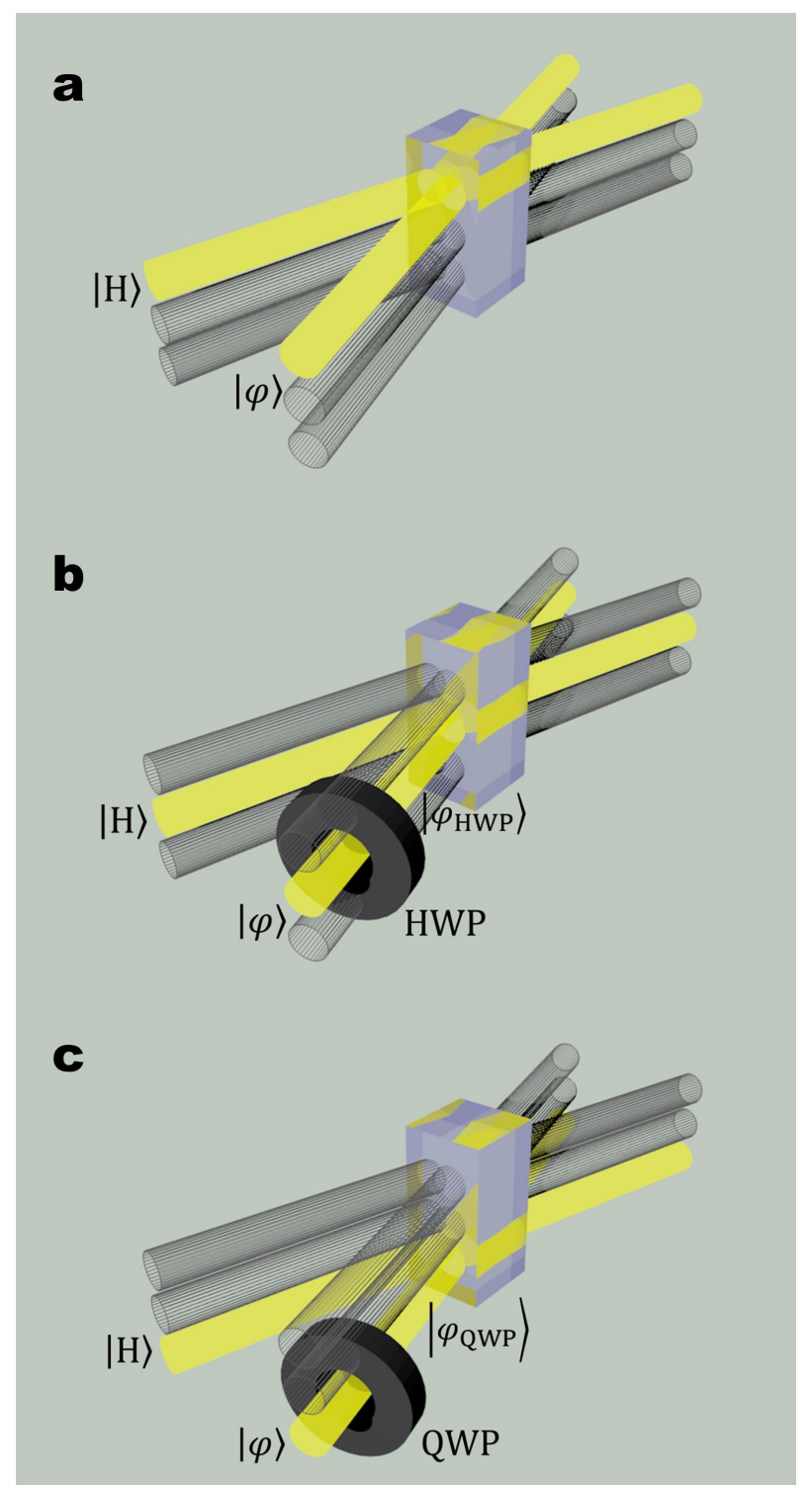

Figure 2. Interference configurations in recording with intensity (a) $I$ and (b) $I_{\mathrm{HWP}}$ and (c) $I_{\mathrm{QWP}}$ 


\subsection{Method of reading a recorded polarization state}

We suppose that the maximum fringe modulation intensity $I_{\max }$ in recording is proportional to the maximum diffraction efficiency $\eta_{\max }$ in reading with the hologram. It is possible to satisfy the condition $\eta_{\max } \propto I_{\max }$ under the specific light intensity and recording time. ${ }^{9,10}$ Under the condition and (7)-(9), the diffraction efficiencies of the beam from top, middle, and bottom position depicted in Figure 2 are given by following equations:

$$
\begin{gathered}
\eta=\eta_{\max } \cos ^{2}\left(\frac{\theta}{2}\right), \\
\eta_{\mathrm{HWP}}=\frac{\eta_{\max }}{2}(1+\cos \phi \sin \theta), \\
\eta_{\mathrm{QWP}}=\frac{\eta_{\max }}{2}(1-\sin \phi \sin \theta) .
\end{gathered}
$$

Refer to the above equations and (2)-(4), the Stokes parameters of the recorded polarization state can be reconstructed with experimentally measured diffraction efficiencies as

$$
\begin{aligned}
& S_{1}=2 \frac{\eta_{\mathrm{HWP}}}{\eta_{\max }}-1, \\
& S_{2}=-2 \frac{\eta_{\mathrm{QWP}}}{\eta_{\max }}+1, \\
& S_{3}=2 \frac{\eta}{\eta_{\max }}-1 .
\end{aligned}
$$

If we previously measure the maximum value of diffraction efficiency $\eta_{\max }$ with the hologram, we can experimentally reconstruct the Stokes parameters of the recorded polarization state by comparing the diffraction efficiencies $\eta, \eta_{\mathrm{HWP}}$, and $\eta_{\mathrm{QWP}}$.

\subsection{Density matrix and Fidelity}

When an arbitrary and pure polarization state is prepared by (1), the theoretically expecting density matrix of the state can be represented by

$$
\rho_{\text {th }} \equiv|\varphi\rangle\langle\varphi|=\frac{1}{2}\left(\begin{array}{cc}
1+\cos \theta & \mathrm{e}^{-i \phi} \sin \theta \\
\mathrm{e}^{i \phi} \sin \theta & 1-\cos \theta
\end{array}\right) .
$$

The density matrix can be also represented by Stokes parameters using the relation (2)-(4), and is given by,

$$
\rho_{\exp }=\frac{1}{2}\left(\begin{array}{cc}
1+S_{3} & S_{1}-i S_{2} \\
S_{1}+i S_{2} & 1-S_{3}
\end{array}\right) .
$$

This equation indicates that the density matrix given by experimentally measured Stokes parameters. In our experiment, we introduce Fidelity, $F=\left\langle\varphi\left|\rho_{\exp }\right| \varphi\right\rangle$, as a measure of overlap between theoretical and experimentally measured polarization states. ${ }^{11,12}$ When the measure is $1(0)$, the theoretical and experimentally measured polarization states are defined as totally identical (orthogonal). Introducing (1) and (17) into the Fidelity, the measure is represented by the theoretically expecting parameters $\theta, \phi$ and experimentally measured Stokes parameters $S_{1}, S_{2}, S_{3}$ as follows:

$$
F=\frac{1}{2}\left\{1+\left(S_{1} \cos \phi+S_{2} \sin \phi\right) \sin \theta+S_{3} \cos \theta\right\} .
$$




\section{EXPERIMENT}

Figure 3 shows the proposed experimental setup. A 532nm diode pumped solid-state cw laser (Millennia V, Spectra-Physics) is converted into a single Gaussian beam through the spatial filter system(SFS). The volume hologram for demonstrating to record arbitrary polarization states is prepared with a representative photorefractive media, $0.015 \%$ iron-doped lithium niobate crystal (Deltronic Crystal). The size of the crystal is 10-mm long , 10-mm wide, and $15-\mathrm{mm}$ high. We record the interference intensity $I, I_{\mathrm{HWP}}, I_{\mathrm{QWP}}$ at three locations 5 -mm apart as shown in Figure 2. POL (polarizer) and HWP0 (half wave plate) are used to adjust the interference intensity and polarization states. The transmitted and reflected beam from PBS (polarization beam splitter) are used as reference and objective beam for the holographic recording. Both of the beams have $30 \mathrm{~mW}$ in intensity and 4-mm FWHM beam diameter. The angle between objective and reflective beams are about 20 degree in air. The polarization state of reference beam is always prepared with horizontal in our experiment. The polarization state of objective beam can be prepared with arbitrary polarization states using HWP1 and QWP1.

In recording, HWP2 and QWP2 work as polarization bases converter as shown HWP and QWP in Fig.2. Using the setup, it is possible to satisfy $\eta_{\max } \propto I_{\max }$ using the general photorefractive material, lithium niobate crystal, with about 30-mW intensity objective and reference beams and about 20-s holographic recording time. In reading process, we block the objective beam with beam dumper (BD), and illuminate the material by a 1 -mW horizontally polarized light from the same direction of the reference beam. We measured the diffraction efficiencies of the three holograms $I, I_{\mathrm{HWP}}, I_{\mathrm{QWP}}$ are recorded 10 times each.

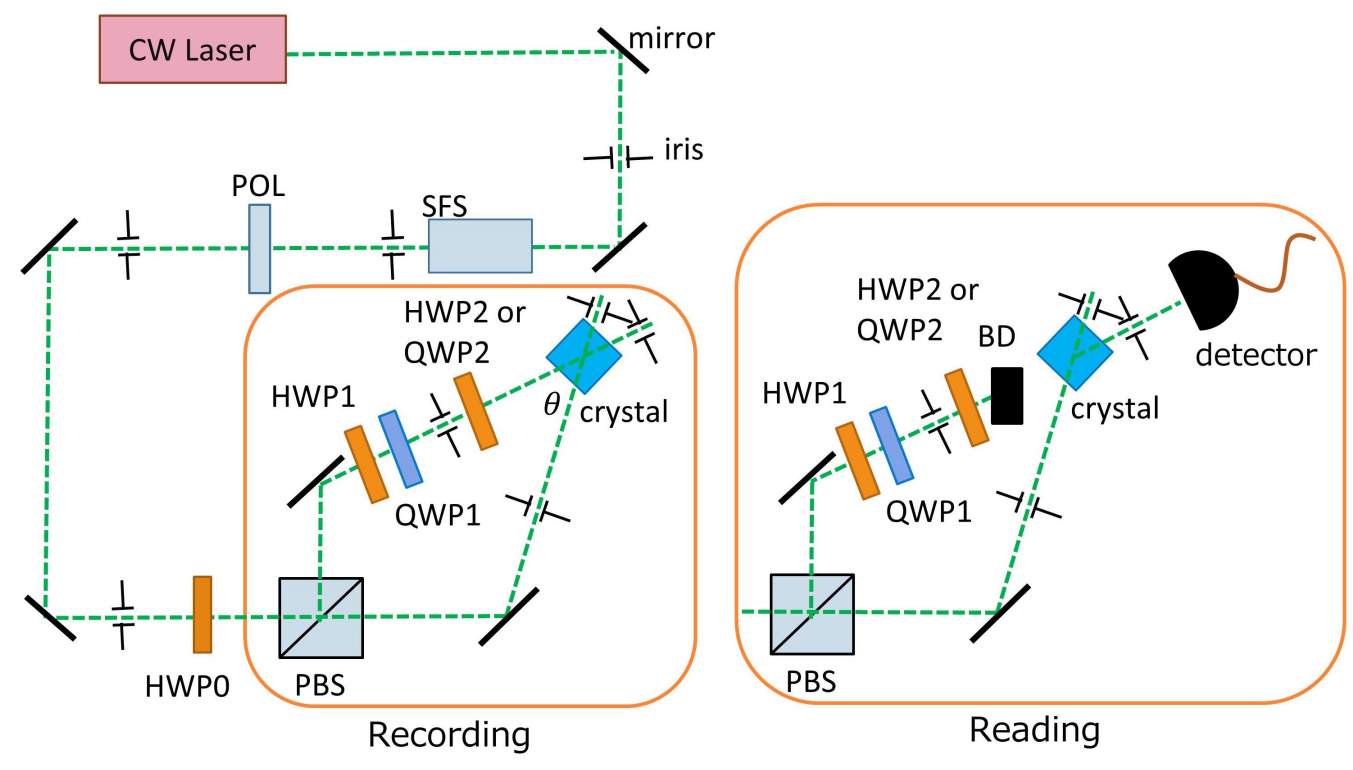

Figure 3. Experimental setup 


\section{SUMMARY}

We proposed a new scheme to record arbitrary polarization states on a general photorefractive media using the relation between the interference intensity in recording process and the diffraction efficiency in reading process. The scheme does not depend on the wavelength of the light nor the material for holographic recording. It is possible to demonstrate the scheme experimentally using a non-polarization dependent photorefractive media, iron-doped lithium niobate crystal.

\section{REFERENCES}

[1] P. Yeh, Introduction to Photorefractive Nonlinear Optics, John Wiley, New York, 1993.

[2] P. J. van Heerden, Theory of Optical Information Storage in Solids, Appl. Opt., 1963, 2, 4, 393-400.

[3] L. Hesselink, and M. C. Bashaw, Optical memories implemented with photorefractive media, Appl. Sci., 1993, 25, 9, S611-S661.

[4] T. Todorov, L. Nikolova, and N. Tomova, Polarization holography. 1: A new high-efficiency organic material with reversible photoinduced birefringence, Appl. Opt., 1984, 23, 23, 4309-4312.

[5] Todorov, L. Nikolova, and N. Tomova, Polarization holography. 2: Polarization holographic gratings in photoanisotropic materials with and without intrinsic birefringence, Appl. Opt., 1984, 23, 24, 4588-4591.

[6] G. R. Fowles, Introduction to Modern Optics, Fourth Edition, Dover Publications, 1989 New York.

[7] E. Hecht, Optics, Addison-Wesley, 2002, San Francisco.

[8] J. B. Altepeter, E. R. Jeffrey, and P. G. Kwiat, Photonic State Tomography, Adv. At. Mol. Opt. Phys., 2005, 52, 105-159.

[9] A. Adibi, K. Buse, and D. Psaltis, Multiplexing holograms in LiNbO3:Fe:Mn crystals, Opt. Lett., 1999, 24, $10,652-654$.

[10] C. Moser, B. Schupp, and D. Psaltis, Localized holographic recording in doubly doped lithium niobate, Opt. Lett., 2000, 25, 3, 162-164.

[11] M. M. Wilde, Quantum Information Theory, Cambridge University Press, 2013, New York.

[12] N. Nielsen and I. Cuang, Quantum Computation and Quantum Information, Cambridge University Press, 2010, New York. 